PROCEEDINGS OF THE

AMERICAN MATHEMATICAL SOCIETY

Volume 138, Number 8, August 2010, Pages 2827-2837

S 0002-9939(10)10321-9

Article electronically published on March 16, 2010

\title{
YANG-TYPE INEQUALITIES FOR WEIGHTED EIGENVALUES OF A SECOND ORDER UNIFORMLY ELLIPTIC OPERATOR WITH A NONNEGATIVE POTENTIAL
}

\author{
HE-JUN SUN
}

(Communicated by Matthew J. Gursky)

\begin{abstract}
In this paper, we investigate the Dirichlet weighted eigenvalue problem of a second order uniformly elliptic operator with a nonnegative potential on a bounded domain $\Omega \subset \mathbb{R}^{n}$. First, we prove a general inequality of eigenvalues for this problem. Then, by using this general inequality, we obtain Yang-type inequalities which give universal upper bounds for eigenvalues. An explicit estimate for the gaps of any two consecutive eigenvalues is also derived. Our results contain and extend the previous results for eigenvalues of the Laplacian, the Schrödinger operator and the second order elliptic operator on a bounded domain $\Omega \subset \mathbb{R}^{n}$.
\end{abstract}

\section{INTRODUCTION}

Let $\Omega \subset \mathbb{R}^{n}$ be a bounded domain with piecewise smooth boundary $\partial \Omega$. Assume that $u(x)$ is a solution of the following Dirichlet eigenvalue problem corresponding to the eigenvalue $\Lambda$ :

$$
\left\{\begin{array}{l}
-\sum_{\substack{i, j=1 \\
u}}^{n} D_{i}\left(a_{i j}(x) D_{j} u\right)=\Lambda u, \quad \text { in } \Omega \subset \mathbb{R}^{n}, \\
\hline\left.\right|_{\partial \Omega},
\end{array}\right.
$$

where $D_{i}=\frac{\partial}{\partial x_{i}}, a_{i j}(x) \in C^{1, \alpha}(\bar{\Omega}), 0<\alpha<1$, and $a_{i j}(x)=a_{j i}(x)$. Moreover, we assume that the coefficients $a_{i j}(x)$ satisfy the uniform ellipticity assumption: for any $\xi=\left(\xi_{1}, \cdots, \xi_{n}\right)$ on every point $x \in \bar{\Omega}$, there is a constant $\varsigma>0$ such that

$$
\sum_{i, j=1}^{n} a_{i j}(x) \xi_{i} \xi_{j} \geq \varsigma|\xi|^{2}
$$

where $|\xi|=\left(\xi_{1}^{2}+\cdots+\xi_{n}^{2}\right)^{\frac{1}{2}}$ denotes the Euclidean norm of $\xi$.

A prototype of a second order elliptic operator $\sum_{i, j=1}^{n} D_{i}\left(a_{i j}(x) D_{j}\right)$ is the Laplacian $\Delta$. When $a_{i j}(x)=\delta_{i j}$, problem (1.1) becomes the classical Dirichlet Laplacian

Received by the editors August 22, 2009, and, in revised form, November 18, 2009.

2010 Mathematics Subject Classification. Primary 35P15, 58C40; Secondary 58J50.

Key words and phrases. Eigenvalue, universal inequality, elliptic operator, Laplacian, Schrödinger operator.

(C)2010 American Mathematical Society Reverts to public domain 28 years from publication 
problem (also called the fixed membrane problem):

$$
\left\{\begin{array}{l}
-\Delta u=\lambda u, \quad \text { in } \Omega \subset \mathbb{R}^{n}, \\
\left.u\right|_{\partial \Omega}=0 .
\end{array}\right.
$$

Let us recall some previous results for eigenvalues of problem (1.3). In 1956, Payne, Pólya and Weinberger [14 considered problem (1.3) and established an important universal inequality for $\Omega \subset \mathbb{R}^{2}$, which was extended to $\Omega \subset \mathbb{R}^{n}$ as the PPW inequality

$$
\lambda_{k+1}-\lambda_{k} \leq \frac{4}{n k} \sum_{t=1}^{k} \lambda_{t} .
$$

The results of Hile and Protter [8], and Yang [19] are two remarkable contributions to the improvements of the PPW inequality. In 1980, Hile and Protter [8] proved

$$
\sum_{t=1}^{k} \frac{\lambda_{t}}{\lambda_{k+1}-\lambda_{t}} \geq \frac{n k}{4}
$$

which is called the HP inequality (cf. 9]). In 1991, Yang [19] obtained Yang's first inequality,

$$
\sum_{t=1}^{k}\left(\lambda_{k+1}-\lambda_{t}\right)^{2} \leq \frac{4}{n} \sum_{t=1}^{k}\left(\lambda_{k+1}-\lambda_{t}\right) \lambda_{t} .
$$

For Yang's original proof, we also refer to [6. Utilizing the Cauchy-Schwarz inequality, one can obtain a weaker inequality, namely Yang's second inequality,

$$
\lambda_{k+1} \leq\left(1+\frac{4}{n}\right) \frac{1}{k} \sum_{t=1}^{k} \lambda_{t} .
$$

Up to now, Yang's first inequality has been the sharpest result for problem (1.3). In 7. Harrell and Stubbe gave a new proof of Yang's inequalities based on commutator algebra. One can find more discussions about inequalities (1.4)-(1.7) in [1, 2] of Ashbaugh. In 2008, Chen and Cheng [3] obtained (1.6) for a bounded domain in an $n$-dimensional complete Riemannian manifold. Some remarkable results for problem (1.3) were also derived on some types of manifolds (see 4, 5, 10, 11, 17, 20 ).

The spectrum of problem (1.1) has been discussed from some angles, but fewer universal inequalities of eigenvalues have been derived. The previous main result was obtained by Qian and Chen [15. They proved

$$
\sum_{t=1}^{k} \frac{\Lambda_{t}}{\Lambda_{k+1}-\Lambda_{t}} \geq \frac{\varsigma n^{2} k}{4}\left(\max _{x \in \bar{\Omega}} \operatorname{tr} A(x)\right)^{-1},
$$

where $\operatorname{tr} A(x)$ denotes the trace of the matrix $A(x)=\left(a_{i j}(x)\right)_{n \times n}$. When $a_{i j}(x)=$ $\delta_{i j}$, it follows that $\operatorname{tr} A(x)=n$. Therefore, (1.8) corresponds to (1.5).

In this paper, we investigate the Dirichlet weighted eigenvalue problem of a second order uniformly elliptic operator with a non-negative potential:

$$
\left\{\begin{array}{l}
-\sum_{i, j=1}^{n} D_{i}\left(a_{i j}(x) D_{j} u\right)+a_{0}(x) u=\Lambda \rho u, \quad \text { in } \Omega \subset \mathbb{R}^{n}, \\
\left.u\right|_{\partial \Omega}=0,
\end{array}\right.
$$


where $a_{0}(x)$ is a non-negative function on $\bar{\Omega}$ and $\rho$ is a positive function continuous on $\bar{\Omega}$. Problem (1.9) is significant in the theory of PDE, the applications of mechanics and physics. In general, the weight function $\rho$ denotes the density. Hence, weighted estimates for eigenvalues have many applications. For example, it is intelligent to make weighted estimates in filtering and identification problems (see [12, 13]).

When $a_{i j}(x)=\delta_{i j}$, problem (1.9) becomes the following weighted eigenvalue problem of the Schrödinger operator:

$$
\left\{\begin{array}{l}
-\Delta u+a_{0}(x) u=\mu \rho u, \quad \text { in } \Omega \subset \mathbb{R}^{n}, \\
\left.u\right|_{\partial \Omega}=0 .
\end{array}\right.
$$

In 2], Ashbaugh obtained Yang's inequalities

$$
\sum_{t=1}^{k}\left(\mu_{k+1}-\mu_{t}\right)^{2} \leq \frac{4 \sigma}{n \tau} \sum_{t=1}^{k}\left(\mu_{k+1}-\mu_{t}\right) \mu_{t}
$$

and

$$
\mu_{k+1} \leq\left(1+\frac{4 \sigma}{n \tau}\right) \frac{1}{k} \sum_{t=1}^{k} \mu_{t} .
$$

For more results of problem (1.10) on the manifolds, we refer the reader to [16, 18].

From the above survey, we find that problem (1.9) synthesizes the eigenvalue problems of various kinds of differential operators, such as the Laplacian, the Schrödinger operator and the second order elliptic operator. It is a very interesting problem.

The goal of this paper is to obtain Yang-type inequalities for the Dirichlet weighted eigenvalues problem (1.9) of a second order uniformly elliptic operator. In Section 2 , we prove a general inequality of eigenvalues. Then, by using this general inequality, we derive Yang-type inequalities for problem (1.9) in Theorem 3.1 and Corollary 3.4. As corollaries of Theorem 3.1, some universal inequalities and bounds of eigenvalues are also derived. For instance, when the potential $a_{0}(x)=0$ and the weight function $\rho=1$, problem (1.9) becomes problem (1.1). As applications of Theorem 3.1, we consider problem (1.1) in Section 4. A series of explicit estimates for problem (1.1) are derived in Corollaries 4.1-4.4. Moreover, our result (4.1) in Corollary 4.1 is sharper than (1.8) derived by Qian and Chen [15] for problem (1.1).

\section{A general ineQuality}

In this section, we shall prove a general inequality for eigenvalues of problem (1.9).

Theorem 2.1. Let $u_{t}$ be the $t$-th weighted orthonormal eigenfunction of problem (1.9) corresponding to the eigenvalue $\Lambda_{t}, t=1,2, \cdots, k$. Namely, $u_{t}$ satisfies

$$
\left\{\begin{array}{l}
-\sum_{i, j=1}^{n} D_{i}\left(a_{i j}(x) D_{j} u_{t}\right)+a_{0}(x) u_{t}=\Lambda_{t} \rho u_{t}, \quad \text { in } \Omega \subset \mathbb{R}^{n}, \\
\left.u_{t}\right|_{\partial \Omega}=0 \\
\int_{\Omega} \rho u_{t} u_{s}=\delta_{t s} .
\end{array}\right.
$$


Then, we have

$$
\begin{aligned}
\sum_{t=1}^{k}\left(\Lambda_{k+1}-\Lambda_{t}\right)^{2} \int_{\Omega} u_{t}^{2} \leq & \delta \sum_{t=1}^{k}\left(\Lambda_{k+1}-\Lambda_{t}\right)^{2} \int_{\Omega} a_{l l}(x) u_{t}^{2} \\
& +\frac{1}{\delta} \sum_{t=1}^{k}\left(\Lambda_{k+1}-\Lambda_{t}\right) \int_{\Omega} \frac{1}{\rho}\left|D_{l} u_{t}\right|^{2},
\end{aligned}
$$

where the constant $\delta>0$.

Proof. Let $x=\left(x_{1}, \cdots, x_{n}\right)$ be the Cartesian coordinate functions of $\mathbb{R}^{n}$. We define the trial functions $\varphi_{t l}$ by

$$
\varphi_{t l}=x_{l} u_{t}-\sum_{s=1}^{k} b_{t s}^{l} u_{s}, \text { for } t=1, \cdots, k \text { and } l=1, \cdots, n,
$$

where

$$
b_{t s}^{l}=\int_{\Omega} \rho x_{l} u_{t} u_{s}=b_{s t}^{l} .
$$

Then, it is easy to check that $\varphi_{t l}$ is weighted orthogonal with $u_{s}$, namely

$$
\int_{\Omega} \rho \varphi_{t l} u_{s}=0, \text { for } t, s=1, \cdots, k .
$$

Hence, it is obvious that

$$
\int_{\Omega} \rho \varphi_{t l} x_{l} u_{t}=\int_{\Omega} \rho \varphi_{t l}^{2} .
$$

By direct calculations, one can get

$$
\begin{aligned}
& \sum_{i, j=1}^{n} D_{i}\left[a_{i j}(x) D_{j}\left(x_{l} u_{t}\right)\right]=\sum_{i=1}^{n} D_{i}\left(a_{i l}(x) u_{t}\right)+\sum_{i, j=1}^{n} D_{i}\left(a_{i j}(x) x_{l} D_{j} u_{t}\right) \\
= & \sum_{i=1}^{n}\left[D_{i}\left(a_{i l}(x) u_{t}\right)+a_{i l}(x) D_{i} u_{t}\right]+x_{l} \sum_{i, j=1}^{n} D_{i}\left(a_{i j}(x) D_{j} u_{t}\right) .
\end{aligned}
$$

This yields

$$
\begin{aligned}
& \sum_{i, j=1}^{n} D_{i}\left(a_{i j}(x) D_{j} \varphi_{t l}\right)-a_{0}(x) \varphi_{t l} \\
= & \sum_{i, j=1}^{n} D_{i}\left[a_{i j}(x) D_{j}\left(x_{l} u_{t}\right)\right]-\sum_{s=1}^{k} b_{t s}^{l} \sum_{i, j=1}^{n} D_{i}\left(a_{i j}(x) D_{j} u_{s}\right)-a_{0}(x) \varphi_{t l} \\
= & \sum_{i=1}^{n}\left[D_{i}\left(a_{i l}(x) u_{t}\right)+a_{i l}(x) D_{i} u_{t}\right]-x_{l}\left[-\sum_{i, j=1}^{n} D_{i}\left(a_{i j}(x) D_{j} u_{t}\right)+a_{0}(x) u_{t}\right] \\
& +\sum_{s=1}^{k} b_{t s}^{l}\left[-\sum_{i, j=1}^{n} D_{i}\left(a_{i j}(x) D_{j} u_{s}\right)+a_{0}(x) u_{s}\right] \\
= & \sum_{i=1}^{n}\left[D_{i}\left(a_{i l}(x) u_{t}\right)+a_{i l}(x) D_{i} u_{t}\right]-x_{l} \Lambda_{t} \rho u_{t}+\sum_{s=1}^{k} b_{t s}^{l} \Lambda_{s} \rho u_{s} .
\end{aligned}
$$


Hence, from (2.5), (2.6) and (2.8), we have

$$
\begin{aligned}
& \int_{\Omega} \varphi_{t l}\left[-\sum_{i, j=1}^{n} D_{i}\left(a_{i j}(x) D_{j} \varphi_{t l}\right)+a_{0}(x) \varphi_{t l}\right] \\
= & -\int_{\Omega} \varphi_{t l} \sum_{i=1}^{n}\left[D_{i}\left(a_{i l}(x) u_{t}\right)+a_{i l}(x) D_{i} u_{t}\right]+\Lambda_{t} \int_{\Omega} \rho \varphi_{t l}^{2} .
\end{aligned}
$$

Substituting (2.9) into the Rayleigh-Ritz formula in the variation method

$$
\Lambda_{k+1} \leq \frac{\int_{\Omega} \varphi_{t l}\left[-\sum_{i, j=1}^{n} D_{i}\left(a_{i j}(x) D_{j} \varphi_{t l}\right)+a_{0}(x) \varphi_{t l}\right]}{\int_{\Omega} \rho \varphi_{t l}^{2}},
$$

we obtain

$$
\begin{aligned}
& \left(\Lambda_{k+1}-\Lambda_{t}\right) \int_{\Omega} \rho \varphi_{t l}^{2} \\
\leq & -\int_{\Omega} \varphi_{t l} \sum_{i=1}^{n}\left[D_{i}\left(a_{i l}(x) u_{t}\right)+a_{i l}(x) D_{i} u_{t}\right] \\
= & -\int_{\Omega} x_{l} u_{t} \sum_{i=1}^{n}\left[D_{i}\left(a_{i l}(x) u_{t}\right)+a_{i l}(x) D_{i} u_{t}\right]+\sum_{s=1}^{k} b_{t s}^{l} c_{t s}^{l},
\end{aligned}
$$

where

$$
c_{t s}^{l}=\int_{\Omega} u_{s} \sum_{i=1}^{n}\left[D_{i}\left(a_{i l}(x) u_{t}\right)+a_{i l}(x) D_{i} u_{t}\right]=-c_{s t}^{l} .
$$

Using integration by parts and utilizing (2.1), we have

$$
\begin{aligned}
\Lambda_{t} b_{t s}^{l}= & \int_{\Omega} x_{l} u_{s}\left[-\sum_{i, j=1}^{n} D_{i}\left(a_{i j}(x) D_{j} u_{t}\right)+a_{0}(x) u_{t}\right] \\
= & -\int_{\Omega} u_{t} \sum_{i, j=1}^{n} D_{j}\left[a_{i j}(x) D_{i}\left(x_{l} u_{s}\right)\right]+\int_{\Omega} a_{0}(x) x_{l} u_{t} u_{s} \\
= & -\int_{\Omega} u_{t} x_{l} \sum_{i, j=1}^{n} D_{j}\left(a_{i j}(x) D_{i} u_{s}\right)-\int_{\Omega} u_{t} \sum_{i=1}^{n} a_{i l}(x) D_{i} u_{s} \\
& -\int_{\Omega} u_{t} \sum_{i=1}^{n} D_{i}\left(a_{i l}(x) u_{s}\right)+\int_{\Omega} a_{0}(x) x_{l} u_{t} u_{s} \\
= & \int_{\Omega} u_{t} x_{l} \Lambda_{s} \rho u_{s}-\int_{\Omega} u_{t} \sum_{i=1}^{n}\left[a_{i l}(x) D_{i} u_{s}+D_{i}\left(a_{i l}(x) u_{s}\right)\right] \\
= & \Lambda_{s} b_{t s}^{l}-c_{s t}^{l} .
\end{aligned}
$$

This yields

$$
c_{t s}^{l}=\left(\Lambda_{t}-\Lambda_{s}\right) b_{t s}^{l}
$$


Moreover, we have

$$
\begin{aligned}
& \int_{\Omega} x_{l} u_{t} \sum_{i=1}^{n}\left[D_{i}\left(a_{i l}(x) u_{t}\right)+a_{i l}(x) D_{i} u_{t}\right] \\
= & \int_{\Omega} x_{l} u_{t} \sum_{i=1}^{n} a_{i l}(x) D_{i} u_{t}-\int_{\Omega} \sum_{i=1}^{n} a_{i l}(x) u_{t} D_{i}\left(x_{l} u_{t}\right) \\
= & \int_{\Omega} x_{l} u_{t} \sum_{i=1}^{n} a_{i l}(x) D_{i} u_{t}-\int_{\Omega} x_{l} u_{t} \sum_{i=1}^{n} a_{i l}(x) D_{i} u_{t}-\int_{\Omega} a_{l l}(x) u_{t}^{2} \\
= & -\int_{\Omega} a_{l l}(x) u_{t}^{2} .
\end{aligned}
$$

Substituting (2.13) and (2.14) into (2.11), we have

$$
\left(\Lambda_{k+1}-\Lambda_{t}\right) \int_{\Omega} \rho \varphi_{t l}^{2} \leq \int_{\Omega} a_{l l}(x) u_{t}^{2}+\sum_{s=1}^{k}\left(\Lambda_{t}-\Lambda_{s}\right)\left(b_{t s}^{l}\right)^{2} .
$$

It is not hard to see that

$$
\int_{\Omega} x_{l} u_{t} D_{l} u_{t}=-\frac{1}{2} \int_{\Omega} u_{t}^{2}
$$

Hence, using integration by parts again, we have

$$
-2 \int_{\Omega} \varphi_{t l} D_{l} u_{t}=-2 \int_{\Omega} x_{l} u_{t} D_{l} u_{t}+2 \sum_{s=1}^{k} b_{t s}^{l} d_{t s}^{l}=\int_{\Omega} u_{t}^{2}+2 \sum_{s=1}^{k} b_{t s}^{l} d_{t s}^{l},
$$

where

$$
d_{t s}^{l}=\int_{\Omega} u_{s} D_{l} u_{t}=-d_{s t}^{l}
$$

On the other hand, we have

$$
\begin{aligned}
& -2\left(\Lambda_{k+1}-\Lambda_{t}\right)^{2} \int_{\Omega} \varphi_{t l} D_{l} u_{t} \\
= & -2\left(\Lambda_{k+1}-\Lambda_{t}\right)^{2} \int_{\Omega} \sqrt{\rho} \varphi_{t l}\left(\frac{1}{\sqrt{\rho}} D_{l} u_{t}-\sqrt{\rho} \sum_{s=1}^{k} d_{t s}^{l} u_{s}\right) \\
\leq & \delta\left(\Lambda_{k+1}-\Lambda_{t}\right)^{3} \int_{\Omega} \rho \varphi_{t l}^{2}+\frac{\Lambda_{k+1}-\Lambda_{t}}{\delta} \int_{\Omega}\left(\frac{1}{\sqrt{\rho}} D_{l} u_{t}-\sqrt{\rho} \sum_{s=1}^{k} d_{t s}^{l} u_{s}\right)^{2} \\
= & \frac{\Lambda_{k+1}-\Lambda_{t}}{\delta}\left[\int_{\Omega} \frac{1}{\rho}\left|D_{l} u_{t}\right|^{2}-2 \sum_{s=1}^{k} d_{t s}^{l} \int_{\Omega} u_{s} D_{l} u_{t}+\sum_{s, q=1}^{k} d_{t s}^{l} d_{t q}^{l} \int_{\Omega} \rho u_{s} u_{q}\right] \\
& +\delta\left(\Lambda_{k+1}-\Lambda_{t}\right)^{3} \int_{\Omega} \rho \varphi_{t l}^{2} \\
= & \delta\left(\Lambda_{k+1}-\Lambda_{t}\right)^{3} \int_{\Omega} \rho \varphi_{t l}^{2}+\frac{\Lambda_{k+1}-\Lambda_{t}}{\delta}\left[\int_{\Omega} \frac{1}{\rho}\left|D_{l} u_{t}\right|^{2}-\sum_{s=1}^{k}\left(d_{t s}^{l}\right)^{2}\right],
\end{aligned}
$$


where $\delta>0$ is a constant to be determined in the proof of Theorem 3.1. Substituting (2.15) into (2.18), we have

$$
\begin{aligned}
& -2\left(\Lambda_{k+1}-\Lambda_{t}\right)^{2} \int_{\Omega} \varphi_{t l} D_{l} u_{t} \\
\leq & \delta\left(\Lambda_{k+1}-\Lambda_{t}\right)^{2}\left[\int_{\Omega} a_{l l}(x) u_{t}^{2}+\sum_{s=1}^{k}\left(\Lambda_{t}-\Lambda_{s}\right)\left(b_{t s}^{l}\right)^{2}\right] \\
& +\frac{\Lambda_{k+1}-\Lambda_{t}}{\delta}\left[\int_{\Omega} \frac{1}{\rho}\left|D_{l} u_{t}\right|^{2}-\sum_{s=1}^{k}\left(d_{t s}^{l}\right)^{2}\right] .
\end{aligned}
$$

Combining (2.17) and (2.19) and taking the sum on $t$ from 1 to $k$, we have

$$
\begin{aligned}
& \sum_{t=1}^{k}\left(\Lambda_{k+1}-\Lambda_{t}\right)^{2} \int_{\Omega} u_{t}^{2}+2 \sum_{t, s=1}^{k}\left(\Lambda_{k+1}-\Lambda_{t}\right)^{2} b_{t s}^{l} d_{t s}^{l} \\
\leq & \delta \sum_{t=1}^{k}\left(\Lambda_{k+1}-\Lambda_{t}\right)^{2} \int_{\Omega} a_{l l}(x) u_{t}^{2}+\delta \sum_{t, s=1}^{k}\left(\Lambda_{k+1}-\Lambda_{t}\right)^{2}\left(\Lambda_{t}-\Lambda_{s}\right)\left(b_{t s}^{l}\right)^{2} \\
& +\frac{1}{\delta} \sum_{t=1}^{k}\left(\Lambda_{k+1}-\Lambda_{t}\right) \int_{\Omega} \frac{1}{\rho}\left|D_{l} u_{t}\right|^{2}-\frac{1}{\delta} \sum_{t, s=1}^{k}\left(\Lambda_{k+1}-\Lambda_{t}\right)\left(d_{t s}^{l}\right)^{2} .
\end{aligned}
$$

Then, we can eliminate the unwanted terms in both sides of (2.20) by using the following equalities:

$$
\sum_{t, s=1}^{k}\left(\Lambda_{k+1}-\Lambda_{t}\right)^{2} b_{t s}^{l} d_{t s}^{l}=-\sum_{t, s=1}^{k}\left(\Lambda_{k+1}-\Lambda_{t}\right)\left(\Lambda_{t}-\Lambda_{s}\right) b_{t s}^{l} d_{t s}^{l}
$$

and

$$
\sum_{t, s=1}^{k}\left(\Lambda_{k+1}-\Lambda_{t}\right)^{2}\left(\Lambda_{t}-\Lambda_{s}\right)\left(b_{t s}^{l}\right)^{2}=-\sum_{t, s=1}^{k}\left(\Lambda_{k+1}-\Lambda_{t}\right)\left(\Lambda_{t}-\Lambda_{s}\right)^{2}\left(b_{t s}^{l}\right)^{2} .
$$

Therefore, (2.2) is true.

\section{YANG-TYPE INEQUALITIES FOR PROBLEM (1.9)}

In this section, we derive Yang-type inequalities for eigenvalues of problem (1.9) by utilizing the general inequality $(2.2)$.

Theorem 3.1. Let $\Lambda_{t}$ be the $t$-th eigenvalue of problem (1.9). Set

$$
\sigma=\left(\min _{x \in \bar{\Omega}} \rho(x)\right)^{-1}, \quad \tau=\left(\max _{x \in \bar{\Omega}} \rho(x)\right)^{-1} .
$$

Assume that $a_{i j}(x)$ satisfies the uniform ellipticity assumption (1.2). Denote by $A(x)$ an $n \times n$ positive definite matrix with components $a_{i j}(x)$ and by $\operatorname{tr} A(x)$ the trace of the matrix $A(x)=\left(a_{i j}(x)\right)_{n \times n}$. Then, we have

$$
\sum_{t=1}^{k}\left(\Gamma_{k+1}-\Gamma_{t}\right)^{2} \leq \frac{4 \sigma^{2}}{n^{2} \tau^{2} \varsigma} \max _{x \in \bar{\Omega}} \operatorname{tr} A(x) \sum_{t=1}^{k}\left(\Gamma_{k+1}-\Gamma_{t}\right) \Gamma_{t}
$$

where $\Gamma_{t}=\Lambda_{t}-\tau \min _{x \in \bar{\Omega}} a_{0}(x)$. 
Proof. Taking the sum on $l$ from 1 to $n$ in (2.2), we have

$$
\begin{aligned}
n \sum_{t=1}^{k}\left(\Lambda_{k+1}-\Lambda_{t}\right)^{2} \int_{\Omega} u_{t}^{2} \leq & \delta \sum_{t=1}^{k}\left(\Lambda_{k+1}-\Lambda_{t}\right)^{2} \int_{\Omega} \sum_{l=1}^{n} a_{l l}(x) u_{t}^{2} \\
& +\frac{1}{\delta} \sum_{t=1}^{k}\left(\Lambda_{k+1}-\Lambda_{t}\right) \int_{\Omega} \frac{1}{\rho} \sum_{l=1}^{n}\left|D_{l} u_{t}\right|^{2} .
\end{aligned}
$$

According to the assumptions of Theorem 3.1, it is not hard to find that

$$
\begin{gathered}
0<\tau=\tau \int_{\Omega} \rho u_{t}^{2} \leq \int_{\Omega} u_{t}^{2}=\int_{\Omega} \rho u_{t}^{2} \frac{1}{\rho} \leq \sigma \int_{\Omega} \rho u_{t}^{2}=\sigma, \\
\sum_{l=1}^{n} \int_{\Omega} a_{l l}(x) u_{t}^{2}=\int_{\Omega} \operatorname{tr} A(x) u_{t}^{2} \leq \sigma \max _{x \in \bar{\Omega}} \operatorname{tr} A(x) .
\end{gathered}
$$

Noticing the uniform ellipticity assumption (1.2), we have

$$
\begin{aligned}
\Lambda_{t} & =\int_{\Omega} u_{t}\left[-\sum_{i, j}^{n} D_{i}\left(a_{i j} D_{j} u_{t}\right)+a_{0}(x) u_{t}\right]=\int_{\Omega} \sum_{i, j}^{n} a_{i j} D_{i} u_{t} D_{j} u_{t}+\int_{\Omega} a_{0}(x) u_{t}^{2} \\
& \geq \varsigma \int_{\Omega}\left|D u_{t}\right|^{2}+\tau \min _{x \in \bar{\Omega}} a_{0}(x) .
\end{aligned}
$$

This yields

$$
\int_{\Omega}\left|D u_{t}\right|^{2} \leq \frac{1}{\varsigma}\left(\Lambda_{t}-\tau \min _{x \in \bar{\Omega}} a_{0}(x)\right)
$$

Therefore, we have

$$
\int_{\Omega} \frac{1}{\rho} \sum_{l=1}^{n}\left|D_{l} u_{t}\right|^{2}=\int_{\Omega} \frac{1}{\rho}\left|D u_{t}\right|^{2} \leq \frac{\sigma}{\varsigma}\left(\Lambda_{t}-\tau \min _{x \in \bar{\Omega}} a_{0}(x)\right) .
$$

Combining (3.3)-(3.6), we obtain

$$
\begin{aligned}
n \tau \sum_{t=1}^{k}\left(\Lambda_{k+1}-\Lambda_{t}\right)^{2} \leq & \delta \sigma \max _{x \in \bar{\Omega}} \operatorname{tr} A(x) \sum_{t=1}^{k}\left(\Lambda_{k+1}-\Lambda_{t}\right)^{2} \\
& +\frac{\sigma}{\delta \varsigma} \sum_{t=1}^{k}\left(\Lambda_{k+1}-\Lambda_{t}\right)\left(\Lambda_{t}-\tau \min _{x \in \bar{\Omega}} a_{0}(x)\right) .
\end{aligned}
$$

Then, putting

$$
\delta=\left[\sum_{t=1}^{k}\left(\Lambda_{k+1}-\Lambda_{t}\right)\left(\Lambda_{t}-\tau \min _{x \in \bar{\Omega}} a_{0}(x)\right)\right]^{\frac{1}{2}}\left[\max _{x \in \bar{\Omega}} \operatorname{tr} A(x) \sum_{t=1}^{k}\left(\Lambda_{k+1}-\Lambda_{t}\right)^{2}\right]^{-\frac{1}{2}}
$$

in (3.7), we have

$$
\begin{aligned}
& n \tau \sum_{t=1}^{k}\left(\Lambda_{k+1}-\Lambda_{t}\right)^{2} \\
\leq & 2 \sigma \varsigma^{-\frac{1}{2}}\left[\sum_{t=1}^{k}\left(\Lambda_{k+1}-\Lambda_{t}\right)^{2}\right]^{\frac{1}{2}}\left[\max _{x \in \bar{\Omega}} \operatorname{tr} A(x) \sum_{t=1}^{k}\left(\Lambda_{k+1}-\Lambda_{t}\right)\left(\Lambda_{t}-\tau \min _{x \in \bar{\Omega}} a_{0}(x)\right)\right]^{\frac{1}{2}} .
\end{aligned}
$$


Deleting $\left[\sum_{t=1}^{k}\left(\Lambda_{k+1}-\Lambda_{t}\right)^{2}\right]^{\frac{1}{2}}$ on both sides of the above inequality yields

$$
\sum_{t=1}^{k}\left(\Lambda_{k+1}-\Lambda_{t}\right)^{2} \leq \frac{4 \sigma^{2}}{n^{2} \tau^{2} \varsigma} \max _{x \in \bar{\Omega}} \operatorname{tr} A(x) \sum_{t=1}^{k}\left(\Lambda_{k+1}-\Lambda_{t}\right)\left(\Lambda_{t}-\tau \min _{x \in \bar{\Omega}} a_{0}(x)\right) .
$$

Then, putting $\Gamma_{t}=\Lambda_{t}-\tau \min _{x \in \bar{\Omega}} a_{0}(x)$ in (3.8), we derive (3.1).

Remark 3.1. We find that the estimate (3.1) depends on the trace of the positive definite matrix $A(x)$. It is well known that $\operatorname{tr} A(x)=\sum_{l=1}^{n} \nu_{l}(x)$, where $\nu_{l}(x)$ is the eigenvalue of the matrix $A(x)$ at $x$, for $l=1, \cdots, n$. Therefore, it is interesting that the coefficient $\max _{x \in \bar{\Omega}} \operatorname{tr} A(x)$ can also be replaced by $\max _{x \in \bar{\Omega}} \sum_{l=1}^{n} \nu_{l}(x)$ or $n \cdot \max _{x \in \bar{\Omega}} \nu_{0}(x)$, where $\nu_{0}(x)$ is the the maximal eigenvalue of $A(x)$ at $x$.

Since $\Gamma_{t}=\Lambda_{t}-\tau \min _{x \in \bar{\Omega}} a_{0}(x) \leq \Lambda_{t}$, we can obtain a weaker inequality:

Corollary 3.2. Under the same assumptions as Theorem 3.1, we have

$$
\sum_{t=1}^{k}\left(\Lambda_{k+1}-\Lambda_{t}\right)^{2} \leq \frac{4 \sigma^{2}}{n^{2} \tau^{2} \varsigma} \max _{x \in \bar{\Omega}} \operatorname{tr} A(x) \sum_{t=1}^{k}\left(\Lambda_{k+1}-\Lambda_{t}\right) \Lambda_{t} .
$$

Noticing that (3.1) is a quadratic inequality of $\Gamma_{k+1}$, we can obtain the following two corollaries which give universal upper bounds of the $(k+1)$-th eigenvalue $\Lambda_{k+1}$.

Corollary 3.3. Under the same assumptions as Theorem 3.1, we have

$$
\begin{aligned}
& \Gamma_{k+1} \leq\left(1+\frac{2 \sigma^{2}}{n^{2} \tau^{2} \varsigma} \max _{x \in \bar{\Omega}}\right.\operatorname{tr} A(x)) \frac{1}{k} \sum_{t=1}^{k} \Gamma_{t}+\left\{\left[\frac{2 \sigma^{2}}{n^{2} \tau^{2} \varsigma} \max _{x \in \bar{\Omega}} \operatorname{tr} A(x) \frac{1}{k} \sum_{t=1}^{k} \Gamma_{t}\right]^{2}\right. \\
&\left.-\left(1+\frac{4 \sigma^{2}}{n^{2} \tau^{2} \varsigma} \max _{x \in \bar{\Omega}} \operatorname{tr} A(x)\right) \frac{1}{k} \sum_{s=1}^{k}\left(\Gamma_{s}-\frac{1}{k} \sum_{t=1}^{k} \Gamma_{t}\right)^{2}\right\}^{\frac{1}{2}} .
\end{aligned}
$$

Corollary 3.4. Under the same assumptions as Theorem 3.1, we have

$$
\Gamma_{k+1} \leq\left(1+\frac{4 \sigma^{2}}{n^{2} \tau^{2} \varsigma} \max _{x \in \bar{\Omega}} \operatorname{tr} A(x)\right) \frac{1}{k} \sum_{t=1}^{k} \Gamma_{t} .
$$

Remark 3.2. The inequalities (3.1) and (3.11) are Yang-type. In fact, it is easy to find that (3.1) and (3.11) respectively correspond to Yang's first inequality (1.6) and Yang's second inequality (1.7).

At the same time, an explicit universal estimate for the gaps of any two consecutive eigenvalues of problem (1.9) can be obtained.

Corollary 3.5. Under the same assumptions as Theorem 3.1, we have

$$
\begin{aligned}
\Lambda_{k+1}-\Lambda_{k} \leq 2\{ & {\left[\frac{2 \sigma^{2}}{n^{2} \tau^{2} \varsigma} \max _{x \in \bar{\Omega}} \operatorname{tr} A(x)\left(\frac{1}{k} \sum_{t=1}^{k} \Lambda_{t}-\tau \min _{x \in \bar{\Omega}} a_{0}(x)\right)\right]^{2} } \\
& \left.-\left(1+\frac{4 \sigma^{2}}{n^{2} \tau^{2} \varsigma} \max _{x \in \bar{\Omega}} \operatorname{tr} A(x)\right) \frac{1}{k} \sum_{s=1}^{k}\left(\Lambda_{s}-\frac{1}{k} \sum_{t=1}^{k} \Lambda_{t}\right)^{2}\right\}^{\frac{1}{2}} .
\end{aligned}
$$




\section{Applications of Theorem 3.1}

In this section, we want to give some applications of Theorem 3.1. One of the reasons for the importance of the estimate (3.1) is that we can easily get some estimates for the special cases of problem (1.9). For example, when the potential $a_{0}(x)=0$ and the weight function $\rho=1$, problem (1.9) becomes problem (1.1). Here, as corollaries of Theorem 3.1, we only give the estimates for eigenvalues of problem (1.1). Of course, the reader can also derive some estimates for other special cases of problem (1.9) from Theorem 3.1.

Corollary 4.1. Let $\Lambda_{t}$ be the $t$-th eigenvalue of problem (1.1). Assume that $\sigma, \tau$ and $A(x)$ satisfy the same assumptions as Theorem 3.1. Then, we have

$$
\sum_{t=1}^{k}\left(\Lambda_{k+1}-\Lambda_{t}\right)^{2} \leq \frac{4}{n^{2} \varsigma} \max _{x \in \bar{\Omega}} \operatorname{tr} A(x) \sum_{t=1}^{k}\left(\Lambda_{k+1}-\Lambda_{t}\right) \Lambda_{t} .
$$

Corollary 4.2. Under the same assumptions as Corollary 4.1, we have

$$
\begin{aligned}
\Lambda_{k+1} \leq & \left(1+\frac{2}{n^{2} \varsigma} \max _{x \in \bar{\Omega}} \operatorname{tr} A(x)\right) \frac{1}{k} \sum_{t=1}^{k} \Lambda_{t}+\left\{\left[\frac{2}{n^{2} \varsigma} \max _{x \in \bar{\Omega}} \operatorname{tr} A(x) \frac{1}{k} \sum_{t=1}^{k} \Lambda_{t}\right]^{2}\right. \\
& \left.-\left(1+\frac{4}{n^{2} \varsigma} \max _{x \in \bar{\Omega}} \operatorname{tr} A(x)\right) \frac{1}{k} \sum_{s=1}^{k}\left(\Lambda_{s}-\frac{1}{k} \sum_{t=1}^{k} \Lambda_{t}\right)^{2}\right\}^{\frac{1}{2}} .
\end{aligned}
$$

Corollary 4.3. Under the same assumptions as Corollary 4.1, we have

$$
\Lambda_{k+1} \leq\left(1+\frac{4}{n^{2} \varsigma} \max _{x \in \bar{\Omega}} \operatorname{tr} A(x)\right) \frac{1}{k} \sum_{t=1}^{k} \Lambda_{t} .
$$

Remark 4.1. When $a_{i j}(x)=\delta_{i j},(4.1)$ and (4.3) respectively become Yang's first inequality (1.6) and Yang's second inequality (1.7). Therefore, our result (4.1) is sharper than (1.8) derived by Qian and Chen [15] for problem (1.1).

Corollary 4.4. Under the same assumptions as Corollary 4.1, we have an estimate for the gaps of any two consecutive eigenvalues of problem (1.1):

$$
\begin{aligned}
\Lambda_{k+1}-\Lambda_{k} \leq 2\{ & {\left[\frac{2}{n^{2} \varsigma} \max _{x \in \bar{\Omega}} \operatorname{tr} A(x) \frac{1}{k} \sum_{t=1}^{k} \Lambda_{t}\right]^{2} } \\
& \left.-\left(1+\frac{4}{n^{2} \varsigma} \max _{x \in \bar{\Omega}} \operatorname{tr} A(x)\right) \frac{1}{k} \sum_{s=1}^{k}\left(\Lambda_{s}-\frac{1}{k} \sum_{t=1}^{k} \Lambda_{t}\right)^{2}\right\}^{\frac{1}{2}} .
\end{aligned}
$$

Remark 4.2. We want to point out that $\Gamma_{t}$ is not the $t$-th eigenvalue of problem (1.9) in Theorem 3.1. If we substitute $\Gamma_{t}=\Lambda_{t}-\tau \min _{x \in \bar{\Omega}} a_{0}(x)$ into the inequalities in Theorem 3.1 and Corollaries 3.3 - 3.5, their actual forms are a little complicated. But for problem (1.1), the situation is different. The above estimates in Corollaries $4.1-4.4$ are very explicit.

\section{ACKNOWLEDGEMENT}

The author would like to express his gratitude to the referee for valuable suggestions. 


\section{REFERENCES}

1. M. S. Ashbaugh, Isoperimetric and universal inequalities for eigenvalues, in Spectral theory and geometry (Edinburgh, 1998), E. B. Davies and Yu Safarov, eds., London Math. Soc. Lecture Notes, Vol. 273, Cambridge Univ. Press, Cambridge, 95-139, 1999. MR.1736867 (2001a:35131)

2. M. S. Ashbaugh, Universal eigenvalue bounds of Payne-Pólya-Weinberger, Hile-Protter and H. C. Yang, Proc. Indian Acad. Sci. (Math. Sci.) 112 (2002), 3-30. MR.1894540 (2004c:35302)

3. D. G. Chen and Q.-M. Cheng, Extrinsic estimates for eigenvalues of the Laplace operator, J. Math. Soc. Japan 60 (2008), 325-339. MR2421979

4. Q.-M. Cheng and H. C. Yang, Estimates on eigenvalues of Laplacian, Math. Ann. 331 (2005), 445-460. MR2115463 (2005i:58038)

5. Q.-M. Cheng and H. C. Yang, Inequalities for eigenvalues of Laplacian on domains and compact complex hypersurfaces in complex projective spaces, J. Math. Soc. Japan 58 (2006), 545-561. MR2228572 (2007k:58051)

6. Q. -M. Cheng and H.C. Yang, Bounds on eigenvalues of Dirichlet Laplacian, Math. Ann. 337 (2007), 159-175. MR2262780 (2007k:35064)

7. E. M. Harrell II and J. Stubbe, On trace identities and universal eigenvalue estimates for some partial differential operators, Trans. Amer. Math. Soc. 349 (1997), 1797-1809. MR1401772 (97i:35129)

8. G. N. Hile and M. H. Protter, Inequalities for eigenvalues of the Laplacian, Indiana Univ. Math. J. 29 (1980), 523-538. MR578204 (82c:35052)

9. S. M. Hook, Domain independent upper bounds for eigenvalues of elliptic operator, Trans. Amer. Math. Soc. 318 (1990), 615-642. MR994167 (90h:35075)

10. P.-F. Leung, On the consecutive eigenvalues of the Laplacain of a compact minimal submanifold in a sphere, J. Austral. Math. Soc. 50 (1991), 409-426. MR1096895 (92d:58212)

11. P. Li, Eigenvalue estimates on homogeneous manifolds, Comment. Math. Helve. 55 (1980), 347-363. MR593051 (81k:58067)

12. L. Ljung, Recursive identification, in Stochastic systems: The mathematics of filtering and identification and applications, M. Hazewinkel and J. C. Willems, eds., Reidel, 1981, 247-283. MR674319 (83m:93001)

13. P. S. Maybeck, Stochastic models, estimation, and control. III, Academic Press, London, 1982. MR0690418 (85a:93134b)

14. L. E. Payne, G. Polya and H. F. Weinberger, On the ratio of consecutive eigenvalues, J. Math. and Phys. 35 (1956), 289-298. MR0084696 (18:905c)

15. C. L. Qian and Z. C. Chen, Estimates of eigenvalues for uniformly elliptic operator of second order, Acta Math. Appli. Sinica 10 (1994), 349-355. MR.1324579 (96b:35158)

16. A. El Soufi, E. M. Harrell II and S. Ilias, Universal inequalities for the eigenvalues of Laplace and Schrödinger operators on submanifolds, Trans. Amer. Math. Soc. 361 (2009), 2337-2350. MR2471921

17. H. J. Sun, Q.-M. Cheng and H. C. Yang, Lower order eigenvalues of Dirichlet Laplacian, Manuscripta Math. 125 (2008), 139-156. MR2373079 (2009i:58042)

18. Q. L. Wang and C. Y. Xia, Universal bounds for eigenvalues of the biharmonic operator on Riemannian manifolds, J. Funct. Anal. 245 (2007), 334-352. MR2311628 (2008e:58033)

19. H. C. Yang, An estimate of the difference between consecutive eigenvalues, preprint IC/91/60, 1991, Trieste, Italy.

20. P. C. Yang and S. T. Yau, Eigenvalues of the Laplacian of compact Riemannian surfaces and minimal submanifolds, Ann. Scuola Norm. Sup. Pisa CI. Sci. 7 (1980), 55-63. MR577325 (81m:58084)

Department of Applied Mathematics, College of Science, Nanjing University of Science and Technology, Nanjing 210094, People's Republic of China

E-mail address: hejunsun@163.com 